\title{
Control chart for waiting time in system of (M / M / S) : ( $\infty$ FCFS) Queuing model
}

\author{
T.Poongodi ${ }^{1}$, Dr. (Mrs.) S.Muthulakshmi ${ }^{2}$ \\ ${ }^{1}$ Assistant Professor, Faculty of Engineering, \\ ${ }^{2}$ Professor, Faculty of Science \\ Avinashilingam Institute for Home Science and Higher Education for Women Coimbatore, India
}

\begin{abstract}
Queuing theory has been applied to a variety of business situations related to customer involvement. The firm provides service facility and tries to keep the costs and time minimum to infuse goodwill among customers. This necessitates the study of service facility to obtain the number of customers and their waiting time. Control chart technique may be applied to analyze the services and the effective performance of concerns. Control chart constructed for the time spent in the system provides the prior idea about expected waiting time, maximum waiting time and minimum waiting time which guarantees customer's satisfaction. Keeping this in view, the construction of control chart for $M / M / s$ queuing model with infinite capacity is proposed in this paper.
\end{abstract}

Keywords -waiting time, control limits, Poisson arrival and exponential service, s- servers.

\section{Introduction}

A common situation that occurs in everyday life is that of queuing or waiting in line. Queues (waiting lines) are usually seen at bus stops, hospitals, bank counters, railway booking counters and so on. In general, queues form when the demand for service exceeds its supply. Waiting time in the facility depends on the number of customers (human being or objects) on queue, the number of servers of serving line and the amount of service time for individual customers. The queuing in relation to the time spent by customers to access services is becoming a major source of concern to service providers. Providing too much of service capacity to operate a system involves loss to service providers whereas not providing enough service capacity results in excessive waiting time and cost to customers. The optimum solution may be obtained by predicting and reducing waiting times and adjusting staffing. Shewhart developed control chart techniques based on data of one or several quality related characteristics of the product or service to identify whether a production process or service having goods of set quality standards.

The analysis of time spent in the system by the control chart technique is suitable since Montgomery [1] proposed a number of applications of control charts in assuring quality in manufacturing industries. Shore [2] developed control chart for random queue length, $\mathrm{N}$ of $\mathrm{M} / \mathrm{M} / 1$ queueing model by considering the first three moments. Khaparde and Dhabe [3] constructed the control chart using method of weighted variance for random queue length $\mathrm{N}$ for $\mathrm{M} / \mathrm{M} / 1$ queueing model. Poongodi and Muthulakshmi [4] analyzed waiting time in system of M/M/1 queuing model using control chart. With reference to individuals sorting for facilities the time spent is more influential than the number in the queuing system. Thus in this paper, an attempt is made to construct Shewhart control chart for waiting time of $\mathrm{M} / \mathrm{M} / \mathrm{s}$ queuing model. This model finds applications in a number of fields where the system is having multiple parallel servers like assembly of machines, aircrafts, ATM centers and supermarkets.

\section{II. $\quad \mathrm{M} / \mathrm{M} / \mathrm{s}$ Queuing Model Description}

$\mathrm{M} / \mathrm{M} / \mathrm{s}$ model has s servers arranged in parallel where the service time at each counter is identical and follows the same exponential law. A customer can go to any of the free counters for his service. The system has infinite capacity with First Come First Serve (FCFS) queue discipline. The customers arrive in a Poisson fashion with mean arrival rate $\lambda$ and the mean service rate $\mu$.

\subsection{Steady state equations}

Let $P_{n}(t)=$ Probability that there are $n$ customers in the system (waiting and in service) at time $t$.

If there are $\mathrm{n}$ customers in the queuing system at any point in time, then the following two cases may arise:

(i) If $\mathrm{n}<\mathrm{s}$, then there will be no queue and $(\mathrm{s}-\mathrm{n})$ number of servers will not be busy. The combined service rate will be $\mu_{\mathrm{n}}=\mathrm{n} \mu, \mathrm{n}<\mathrm{s}$.

(ii) If $\mathrm{n} \geq \mathrm{s}$, then all the servers will be busy and the maximum number of customers in the queue will be $(\mathrm{n}-\mathrm{s})$. Then $\mu_{\mathrm{n}}=\mathrm{s} \mu, \mathrm{n} \geq \mathrm{s}$. 
The governing equations of this model are

$$
\begin{aligned}
& \text { The governing equations of this model are } \\
& \qquad \begin{array}{l}
\mathrm{P}_{0}(\mathrm{t}+\Delta \mathrm{t})=\mathrm{P}_{0}(\mathrm{t})(1-\lambda \Delta \mathrm{t})+\mathrm{P}_{1}(\mathrm{t}) \mu \Delta \mathrm{t}+\mathrm{o}(\Delta \mathrm{t}) \mathrm{n}=0 \\
\mathrm{P}_{\mathrm{n}}(\mathrm{t}+\Delta \mathrm{t})=\mathrm{P}_{\mathrm{n}}(\mathrm{t})(1-(\lambda+\mathrm{n} \mu) \Delta \mathrm{t})+\mathrm{P}_{\mathrm{n}-1}(\mathrm{t}) \lambda \Delta \mathrm{t}+\mathrm{P}_{\mathrm{n}+1}(\mathrm{t})(\mathrm{n}+1) \mu \Delta \mathrm{t}+\mathrm{o}(\Delta \mathrm{t}), \mathrm{n}<\mathrm{s}
\end{array} \\
& \text { and } \quad \begin{array}{r}
\mathrm{P}_{\mathrm{n}}(\mathrm{t}+\Delta \mathrm{t})=\mathrm{P}_{\mathrm{n}}(\mathrm{t})(1-(\lambda+\mathrm{s} \mu) \Delta \mathrm{t})+\mathrm{P}_{\mathrm{n}-1}(\mathrm{t}) \lambda \Delta \mathrm{t}+\mathrm{P}_{\mathrm{n}+1}(\mathrm{t}) \mathrm{s} \mu \Delta \mathrm{t}+\mathrm{o}(\Delta \mathrm{t}), \mathrm{n} \geq \mathrm{s} \\
\text { Equation }
\end{array}
\end{aligned}
$$

Equation (1) gives

$$
\begin{gathered}
\mathrm{P}_{0}^{\prime}(\mathrm{t})=-\lambda \mathrm{P}_{0}(\mathrm{t})+\mu \mathrm{P}_{1}(\mathrm{t}), \mathrm{n}=0 \\
\mathrm{P}_{\mathrm{n}}^{\prime}(\mathrm{t})=-(\lambda+\mathrm{n} \mu) \mathrm{P}_{\mathrm{n}}(\mathrm{t})+\lambda \mathrm{P}_{\mathrm{n}-1}(\mathrm{t})+(\mathrm{n}+1) \mu \mathrm{P}_{\mathrm{n}+1}(\mathrm{t}), \mathrm{n}<\mathrm{s}
\end{gathered}
$$

and $\quad \mathrm{P}_{\mathrm{n}}{ }^{\prime}(\mathrm{t})=-(\lambda+\mathrm{s} \mu) \mathrm{P}_{\mathrm{n}}(\mathrm{t})+\lambda \mathrm{P}_{\mathrm{n}-1}(\mathrm{t})+\mathrm{s} \mu \mathrm{P}_{\mathrm{n}+1}(\mathrm{t}), \quad \mathrm{n} \geq \mathrm{s}$

The steady state equations corresponding to (2) are

$$
\begin{aligned}
& 0=-\lambda P_{0}+\mu P_{1}, n=0 \\
& 0=-(\lambda+n \mu) P_{n}+\lambda P_{n-1}+(n+1) \mu P_{n+1}, n<s \\
& \text { and } \quad 0=-(\lambda+s \mu) P_{n}+\lambda P_{n-1}+s \mu P_{n+1}, \quad n \geq s
\end{aligned}
$$

Let $\rho=\lambda / s \mu$ be the traffic intensity. From equation (3)

$$
\mathrm{P}_{\mathrm{n}}=\left\{\begin{array}{l}
\frac{1}{\mathrm{n} !}(\lambda / \mu){ }^{\mathrm{n}} \mathrm{P}_{0}, \mathrm{n}<\mathrm{s} \\
\frac{1}{\mathrm{~s}^{\mathrm{n}}}\left(1 / \mathrm{s}^{\mathrm{n}-\mathrm{s}}\right)(\lambda / \mu){ }^{\mathrm{n}} \mathrm{P}_{0}, \mathrm{n} \geq \mathrm{s}
\end{array}\right.
$$

where

$$
\mathrm{P}_{0}=\left\{\sum_{n=0}^{s-1} \frac{(\mathrm{s} \rho)^{\mathrm{n}}}{\mathrm{n} !}+\frac{(\rho \mathrm{s})^{\mathrm{s}}}{\mathrm{s} !(1-\rho)}\right\}^{-1}
$$

\subsection{Performance measures}

(i) $\mathrm{E}\left(\mathrm{L}_{\mathrm{q}}\right)=$ Average number of customers in the queue

$$
\begin{aligned}
& =\sum_{n=s}^{\infty}(n-s) P_{0} \\
& =\frac{\rho(\rho s)^{s}}{s !(1-\rho)^{2}} P_{0}
\end{aligned}
$$

(ii) $\mathrm{E}\left(\mathrm{L}_{\mathrm{s}}\right)=$ Average number of customers in the system

$$
=\frac{\rho(\rho s)^{s}}{s !(1-\rho)^{2}} P_{0}+\frac{\lambda}{\mu}
$$

(iii) $\mathrm{E}\left(\mathrm{W}_{\mathrm{q}}\right)=$ Average waiting time of a customer in the queue

$$
=\frac{(s \rho)^{s}}{s ! s \mu(1-\rho)^{2}} P_{0}
$$

(iv) $\mathrm{E}(\mathrm{W})=$ Average waiting time of a customer in the system

$$
=\frac{(s \rho)^{s}}{s ! s \mu(1-\rho)^{2}} P_{0}+\frac{1}{\mu}
$$

\section{Control Chart for Waiting time, W}

Let $\mathrm{W}$ denote the waiting time of a customer in the system which includes both the waiting time and the service time. The pdf of the random variable $\mathrm{W}$ as in Gross and Harris [5] is 


$$
\begin{aligned}
& f(t)=\frac{1}{(s-s \rho-1)}\left[\left(s(1-\rho)-1+\frac{(\rho s)^{s}}{s !(1-\rho)} P_{0}\right) \mu e^{-\mu t}-\frac{(\rho s)^{s}}{s !(1-\rho)} P_{0}(s \mu-\lambda) e^{-(s \mu-\lambda) t}\right], t>0 \\
& . E(W)=\frac{(s \rho)^{s}}{s ! s \mu(1-\rho)^{2}} P_{0}+\frac{1}{\mu} \text { and } \\
& \operatorname{Var}(W)=\frac{1}{\mu^{2}}\left[2\left(1+\frac{(\rho s)^{s}(\mu(s+1)-\lambda)}{\mu s !^{2}(1-\rho)^{3}} P_{0}\right)-\left(1+\frac{(\rho s)^{s}}{s ! s(1-\rho)^{2}} P_{0}\right)^{2}\right]
\end{aligned}
$$

The parameters of Shewhart type control charts, by approximating the statistic under consideration by a normal distribution, are given by

$$
\left.\begin{array}{l}
\begin{array}{l}
\text { distribution, are given by } \\
\mathrm{UCL}
\end{array}=\mathrm{E}(\mathrm{W})+3 \sqrt{\mathrm{V}(\mathrm{W})} \\
\mathrm{CL} \quad=\mathrm{E}(\mathrm{W}) \\
\mathrm{LCL} \quad=\mathrm{E}(\mathrm{W})-3 \sqrt{\mathrm{V}(\mathrm{W})}
\end{array}\right\}
$$

For $\mathrm{M} / \mathrm{M} / \mathrm{s}$ queuing model the parameters of the control chart for waiting time of the customer in the system are given by

$$
\begin{aligned}
\mathrm{UCL} & =\frac{1}{\mu}\left[\left(1+\frac{(\rho \mathrm{s})^{\mathrm{s}}}{\mathrm{s} ! \mathrm{s}(1-\rho)^{2}} \mathrm{P}_{0}\right)+3\left[2\left(1+\frac{(\rho \mathrm{s})^{\mathrm{s}}(\mathrm{s}+1-\rho)}{\mathrm{s} \mathrm{s}^{2}(1-\rho)^{3}} \mathrm{P}_{0}\right)-\left(1+\frac{(\rho \mathrm{s})^{\mathrm{s}}}{\mathrm{s} ! \mathrm{s}(1-\rho)^{2}} \mathrm{P}_{0}\right)^{2}\right]^{1 / 2}\right] \\
\mathrm{CL} & =\frac{(\mathrm{s} \rho)^{\mathrm{s}}}{\mathrm{s} ! \mathrm{s} \mu(1-\rho)^{2}} \mathrm{P}_{0}+\frac{1}{\mu} \\
\mathrm{LCL} & =\frac{1}{\mu}\left[\left(1+\frac{(\rho s)^{\mathrm{s}}}{\mathrm{s} ! \mathrm{s}(1-\rho)^{2}} \mathrm{P}_{0}\right)-3\left[2\left(1+\frac{(\rho s)^{\mathrm{s}}(\mathrm{s}+1-\rho)}{\mathrm{s} \mathrm{s}^{2}(1-\rho)^{3}} \mathrm{P}_{0}\right)-\left(1+\frac{(\rho s)^{\mathrm{s}}}{\mathrm{s} ! \mathrm{s}(1-\rho)^{2}} \mathrm{P}_{0}\right)^{2}\right]^{1 / 2}\right]
\end{aligned}
$$

\section{Numerical Analysis}

Assessment of waiting time in the system by means of control chart is carried out with numerical illustrations for certain selected values of $\lambda$ and $\mu$. Tables1, 2 and 3 give the parameters of the control chart for various values of the arrival rate $\lambda$ and a constant service rate $\mu=6$ and the number of servers $s=2,4$ and 6 respectively. 
Table.1. Parameters of control chart for $\mu=6$ and $s=2$

\begin{tabular}{|c|c|c|c|c|c|}
\hline$\lambda$ & $\mu$ & $\rho$ & $\mathrm{s}$ & $\mathrm{CL}$ & $\mathrm{UCL}$ \\
\hline 1.00 & 6 & 0.0833 & 2 & 0.1678 & 4.4084 \\
\hline 1.50 & 6 & 0.1250 & 2 & 0.1693 & 4.4525 \\
\hline 2.00 & 6 & 0.1667 & 2 & 0.1714 & 4.5257 \\
\hline 2.50 & 6 & 0.2083 & 2 & 0.1742 & 4.6378 \\
\hline 3.00 & 6 & 0.2500 & 2 & 0.1778 & 4.8019 \\
\hline 3.50 & 6 & 0.2917 & 2 & 0.1822 & 5.0338 \\
\hline 4.00 & 6 & 0.3333 & 2 & 0.1875 & 5.3528 \\
\hline 4.50 & 6 & 0.3750 & 2 & 0.1939 & 5.7858 \\
\hline 5.00 & 6 & 0.4167 & 2 & 0.2017 & 6.3641 \\
\hline 5.50 & 6 & 0.4583 & 2 & 0.2110 & 7.1266 \\
\hline 6.00 & 6 & 0.5000 & 2 & 0.2222 & 8.1314 \\
\hline 6.50 & 6 & 0.5417 & 2 & 0.2359 & 9.4524 \\
\hline 7.00 & 6 & 0.5833 & 2 & 0.2526 & 11.1935 \\
\hline 7.50 & 6 & 0.6250 & 2 & 0.2735 & 13.5272 \\
\hline 8.00 & 6 & 0.6667 & 2 & 0.3000 & 16.7100 \\
\hline
\end{tabular}

Table.2. Parameters of control chart for $\mu=6$ and $s=4$

\begin{tabular}{|c|c|c|c|c|c|}
\hline$\lambda$ & $\mu$ & $\rho$ & $\mathrm{s}$ & $\mathrm{CL}$ & $\mathrm{UCL}$ \\
\hline 1.00 & 6 & 0.0417 & 4 & 0.1667 & 4.3798 \\
\hline 1.50 & 6 & 0.0625 & 4 & 0.1667 & 4.3799 \\
\hline 2.00 & 6 & 0.0833 & 4 & 0.1667 & 4.3802 \\
\hline 2.50 & 6 & 0.1042 & 4 & 0.1667 & 4.3809 \\
\hline 3.00 & 6 & 0.1250 & 4 & 0.1668 & 4.3824 \\
\hline 3.50 & 6 & 0.1458 & 4 & 0.1668 & 4.3848 \\
\hline 4.00 & 6 & 0.1667 & 4 & 0.1669 & 4.3891 \\
\hline 4.50 & 6 & 0.1875 & 4 & 0.1671 & 4.3958 \\
\hline 5.00 & 6 & 0.2083 & 4 & 0.1672 & 4.4059 \\
\hline 5.50 & 6 & 0.2292 & 4 & 0.1675 & 4.4210 \\
\hline 6.00 & 6 & 0.2500 & 4 & 0.1678 & 4.4427 \\
\hline 6.50 & 6 & 0.2708 & 4 & 0.1682 & 4.4731 \\
\hline 7.00 & 6 & 0.2917 & 4 & 0.1687 & 4.5155 \\
\hline 7.50 & 6 & 0.3125 & 4 & 0.1692 & 4.5730 \\
\hline 8.00 & 6 & 0.3333 & 4 & 0.1699 & 4.6501 \\
\hline
\end{tabular}

Table.3. Parameters of control chart for $\mu=6$ and $s=6$

\begin{tabular}{|c|c|c|c|c|c|}
\hline$\lambda$ & $\mu$ & $\rho$ & $\mathrm{s}$ & $\mathrm{CL}$ & $\mathrm{UCL}$ \\
\hline 1.00 & 6 & 0.0278 & 6 & 0.1667 & 4.3798 \\
\hline 1.50 & 6 & 0.0417 & 6 & 0.1667 & 4.3798 \\
\hline 2.00 & 6 & 0.0556 & 6 & 0.1667 & 4.3798 \\
\hline 2.50 & 6 & 0.0694 & 6 & 0.1667 & 4.3798 \\
\hline 3.00 & 6 & 0.0833 & 6 & 0.1667 & 4.3798 \\
\hline 3.50 & 6 & 0.0972 & 6 & 0.1667 & 4.3798 \\
\hline 4.00 & 6 & 0.1111 & 6 & 0.1667 & 4.3798 \\
\hline
\end{tabular}




\begin{tabular}{|l|l|l|l|l|l|}
4.50 & 6 & 0.1250 & 6 & 0.1667 & 4.3799 \\
\hline 5.00 & 6 & 0.1389 & 6 & 0.1667 & 4.3801 \\
\hline 5.50 & 6 & 0.1528 & 6 & 0.1667 & 4.3803 \\
\hline 6.00 & 6 & 0.1667 & 6 & 0.1667 & 4.3807 \\
\hline 6.50 & 6 & 0.1806 & 6 & 0.1667 & 4.3814 \\
\hline 7.00 & 6 & 0.1944 & 6 & 0.1667 & 4.3825 \\
\hline 7.50 & 6 & 0.2083 & 6 & 0.1667 & 4.3841 \\
\hline 8.00 & 6 & 0.2222 & 6 & 0.1668 & 4.3865 \\
\hline
\end{tabular}

Numerical values of Tables 1, 2 and 3 reveal the following facts:

(i) increase in arrival rate with constant service rate increases the average waiting time and the expected upper limit of waiting time.

(ii) increase in number of servers decreases the average waiting time and expected maximum waiting time.

Tables 4, 5 and 6 give the parameters of the control chart for a constant arrival rate $\lambda$ and various values of the service rate $\mu$ and the number of servers $s=2,4$ and 6 respectively.

Table.4. Parameters of control chart for $\lambda=4$ and $s=2$

\begin{tabular}{|c|c|c|c|c|r|}
\hline$\lambda$ & $\mu$ & $\rho$ & $\mathrm{s}$ & $\mathrm{CL}$ & \multicolumn{1}{c|}{ UCL } \\
\hline 4 & 2.25 & 0.8889 & 2 & 2.1174 & 106.6408 \\
\hline 4 & 2.50 & 0.8000 & 2 & 1.1110 & 40.9970 \\
\hline 4 & 2.75 & 0.7273 & 2 & 0.7721 & 24.1422 \\
\hline 4 & 3.00 & 0.6667 & 2 & 0.6001 & 16.9358 \\
\hline 4 & 3.25 & 0.6154 & 2 & 0.4953 & 13.0915 \\
\hline 4 & 3.50 & 0.5714 & 2 & 0.4242 & 10.7693 \\
\hline 4 & 3.75 & 0.5333 & 2 & 0.3726 & 9.2532 \\
\hline 4 & 4.00 & 0.5000 & 2 & 0.3333 & 8.2073 \\
\hline 4 & 4.25 & 0.4706 & 2 & 0.3022 & 7.4546 \\
\hline 4 & 4.50 & 0.4444 & 2 & 0.2769 & 6.8947 \\
\hline 4 & 4.75 & 0.4211 & 2 & 0.2559 & 6.4708 \\
\hline 4 & 5.00 & 0.4000 & 2 & 0.2381 & 6.1392 \\
\hline 4 & 5.25 & 0.3810 & 2 & 0.2228 & 5.8778 \\
\hline 4 & 5.50 & 0.3636 & 2 & 0.2095 & 5.6662 \\
\hline 4 & 5.75 & 0.3478 & 2 & 0.1978 & 5.4946 \\
\hline
\end{tabular}

Table.5. Parameters of control chart for $\lambda=4$ and $s=4$

\begin{tabular}{|c|c|c|c|c|c|}
\hline$\lambda$ & $\mu$ & $\rho$ & $\mathrm{s}$ & $\mathrm{CL}$ & $\mathrm{UCL}$ \\
\hline 4 & 2.00 & 0.5000 & 4 & 0.5435 & 7.0505 \\
\hline 4 & 2.25 & 0.4444 & 4 & 0.4692 & 5.8031 \\
\hline 4 & 2.50 & 0.4000 & 4 & 0.4151 & 5.2100 \\
\hline 4 & 2.75 & 0.3636 & 4 & 0.3733 & 4.9031 \\
\hline 4 & 3.00 & 0.3333 & 4 & 0.3398 & 4.7322 \\
\hline 4 & 3.25 & 0.3077 & 4 & 0.3122 & 4.6301 \\
\hline 4 & 3.50 & 0.2857 & 4 & 0.2889 & 4.5648 \\
\hline 4 & 3.75 & 0.2667 & 4 & 0.2690 & 4.5208 \\
\hline 4 & 4.00 & 0.2500 & 4 & 0.2517 & 4.4893 \\
\hline 4 & 4.25 & 0.2353 & 4 & 0.2366 & 4.4660 \\
\hline 4 & 4.50 & 0.2222 & 4 & 0.2232 & 4.4480 \\
\hline 4 & 4.75 & 0.2105 & 4 & 0.2113 & 4.4336 \\
\hline
\end{tabular}




\begin{tabular}{|l|l|l|l|l|l|}
4 & 5.00 & 0.2000 & 4 & 0.2006 & 4.4217 \\
\hline 4 & 5.25 & 0.1905 & 4 & 0.1910 & 4.4118 \\
\hline 4 & 5.50 & 0.1818 & 4 & 0.1822 & 4.4032 \\
\hline
\end{tabular}

Table.6. Parameters of control chart for

\begin{tabular}{|c|c|c|c|c|c|}
\hline$\lambda$ & $\mu$ & $\rho$ & $\mathrm{s}$ & $\mathrm{CL}$ & $\mathrm{UCL}$ \\
\hline 4 & 2.00 & 0.3333 & 6 & 0.5023 & 4.5923 \\
\hline 4 & 2.25 & 0.2963 & 6 & 0.4456 & 4.5242 \\
\hline 4 & 2.50 & 0.2667 & 6 & 0.4006 & 4.4939 \\
\hline 4 & 2.75 & 0.2424 & 6 & 0.3640 & 4.4761 \\
\hline 4 & 3.00 & 0.2222 & 6 & 0.3335 & 4.4633 \\
\hline 4 & 3.25 & 0.2051 & 6 & 0.3078 & 4.4527 \\
\hline 4 & 3.50 & 0.1905 & 6 & 0.2858 & 4.4433 \\
\hline 4 & 3.75 & 0.1778 & 6 & 0.2667 & 4.4347 \\
\hline 4 & 4.00 & 0.1667 & 6 & 0.2500 & 4.4268 \\
\hline 4 & 4.25 & 0.1569 & 6 & 0.2353 & 4.4195 \\
\hline 4 & 4.50 & 0.1481 & 6 & 0.2222 & 4.4126 \\
\hline 4 & 4.75 & 0.1404 & 6 & 0.2105 & 4.4062 \\
\hline 4 & 5.00 & 0.1333 & 6 & 0.2000 & 4.4002 \\
\hline 4 & 5.25 & 0.1270 & 6 & 0.1905 & 4.3946 \\
\hline 4 & 5.50 & 0.1212 & 6 & 0.1818 & 4.3894 \\
\hline
\end{tabular}

Tables 4, 5 and 6 reveal the following facts:

(i) increase in service rate with constant arrival rate decreases the average waiting time and the expected upper limit of waiting time.

(ii) increase in number of servers decreases the average waiting time and expected maximum waiting time.

\section{References}

[1] Montgomery D.C., Introduction to statistical quality control ( $5^{\text {th }}$ Edition John Wiley \& Sons, Inc , 2005)

[2] Shore,H. General control charts for attributes, IIE transactions, 32, 2000, 1149-1160.

[3] Khaparde, M.V. and Dhabe, S.D. Control chart for random queue length N for (M/M/1):( $\infty / F C F S)$ queueing model,International Journal of Agricultural and Statistical sciences, Vol.1, 2010, 319-334.

[4] Poongodi.T and Muthulakshmi. S. (2013), Control chart for waiting time in system of (M/M/1): ( $\infty / F C F S)$ Queueing model, International Journal of Computer Applications, Vol 63, No.3, 2013, 1 - 6.

[5] Gross, D. and Harris, C.M., Fundamentals of queueing theory ( $3^{\text {rd }}$ edition, (1998), John Wiley and sons. 